\title{
Identification and Investigation of Autolysin Genes in Clostridium saccharoperbutylacetonicum Strain N1-4 for Enhanced Biobutanol Production
}

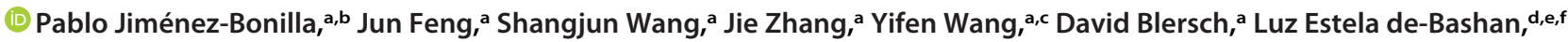 \\ Philippe Gaillard, ${ }^{9}$ Liang Guo, ${ }^{\text {h }}$ (D) Yi Wanga,c
}

\author{
aDepartment of Biosystems Engineering, Auburn University, Auburn, Alabama, USA \\ bSchool of Chemistry, National University (UNA), Heredia, Costa Rica \\ ‘Center for Bioenergy and Bioproducts, Auburn University, Auburn, Alabama, USA \\ dEnvironmental Microbiology Group, Northwestern Center for Biological Research (CIBNOR), La Paz, Mexico \\ eThe Bashan Institute of Science, Auburn, Alabama, USA \\ fDepartment of Entomology and Plant Pathology, Auburn University, Auburn, Alabama, USA \\ gStatistical Consulting Center, Mathematics and Statistics Department, Auburn University, Auburn, Alabama, USA \\ ${ }^{n}$ College of Environmental Science and Engineering, Ocean University of China, Qingdao, China
}

ABSTRACT Biobutanol is a valuable biochemical and one of the most promising biofuels. Clostridium saccharoperbutylacetonicum N1-4 is a hyperbutanol-producing strain. However, its strong autolytic behavior leads to poor cell stability, especially during continuous fermentation, thus limiting the applicability of the strain for longterm and industrial-scale processes. In this study, we aimed to evaluate the role of autolysin genes within the C. saccharoperbutylacetonicum genome related to cell autolysis and further develop more stable strains for enhanced butanol production. First, putative autolysin-encoding genes were identified in the strain based on comparison of amino acid sequence with homologous genes in other strains. Then, by overexpressing all these putative autolysin genes individually and characterizing the corresponding recombinant strains, four key genes were pinpointed to be responsible for significant cell autolysis activities. Further, these key genes were deleted using CRISPR-Cas9. Fermentation characterization demonstrated enhanced performance of the resultant mutants. Results from this study reveal valuable insights concerning the role of autolysins for cell stability and solvent production, and they provide an essential reference for developing robust strains for enhanced biofuel and biochemical production.

IMPORTANCE Severe autolytic behavior is a common issue in Clostridium and many other microorganisms. This study revealed the key genes responsible for the cell autolysis within Clostridium saccharoperbutylacetonicum, a prominent platform for biosolvent production from lignocellulosic materials. The knowledge generated in this study provides insights concerning cell autolysis in relevant microbial systems and gives essential references for enhancing strain stability through rational genome engineering.

KEYWORDS autolysis, Clostridium, biobutanol, fermentation, CRISPR-Cas9, biofuel

iobutanol ( $n$-butanol) produced from low-value renewable resources through fermentation with solventogenic clostridia attracts great interest as a potential biofuel source and biochemical feedstock with various applications (1). However, currently, there are still various limitations for biobutanol production, which often lead to low titer, yield, and productivity and prevent the process from being economically
Citation Jiménez-Bonilla P, Feng J, Wang S, Zhang J, Wang Y, Blersch D, de-Bashan LE, Gaillard P, Guo L, Wang Y. 2021. Identification and investigation of autolysin genes in Clostridium saccharoperbutylacetonicum strain N1-4 for enhanced biobutanol production. App Environ Microbiol 87:e02442-20. https://doi.org/ 10.1128/AEM.02442-20.

Editor Shuang-Jiang Liu, Chinese Academy of Sciences

Copyright $\odot 2021$ American Society for Microbiology. All Rights Reserved. Address correspondence to Yi Wang, yiwang3@auburn.edu.

Received 5 October 2020 Accepted 14 January 2021 Accepted manuscript posted online 29 January 2021

Published 11 March 2021 
competitive. Clostridium saccharoperbutylacetonicum N1-4 is a hyperbutanol-producing strain, which can produce much higher butanol levels than other well-known clostridial type strains (including C. beijerinckii NCIMB 8052 and C. acetobutylicum ATCC 824) in regular batch fermentations under similar conditions (2-4). However, it is often observed that the cells of $C$. saccharoperbutylacetonicum N1-4 experienced severe cell lysis at the end of the fermentation. In addition, C. saccharoperbutylacetonicum N1-4 often has unstable performance for acetone, butanol, and ethanol (ABE) production during continuous fermentation.

Autolysis activity is known in many Clostridium species primarily due to the existence of autolysins. They are responsible for the hydrolysis of different components in the cell wall; based on the different hydrolytic activity on different functional groups of the peptidoglycan, the autolysins are classified into different groups (5). They also play roles in processes such as cell motility, cell separation, cell elongation, peptidoglycan maturation, cell wall turnover, germination, sporulation, and induced lysis (6). Spontaneous autolysis leads to significant loss of cell biomass, and no obvious stationary phase could be observed during the cell growth of solventogenic clostridia (7). Induced autolysis (accompanied by sporulation) could also occur due to chemical-induced stresses, such as stresses from oxygen, acetate, butyrate, and butanol (8).

Autolysins genes have been reported in several solventogenic clostridial strains, such as lyt-1 (from C. acetobutylicum P262), CA_C0554 (from C. acetobutylicum ATCC 824), (9, 10), SMB_G2359, and SMB_G3117 (from C. acetobutylicum DSM 1731) (5). CA_C0554 was reported to play an important role in sporulation (7), and the disruption of SMB_G3117 led to a significant increase in the cell biomass (5). Some $N$-acetylmuramidases have also been isolated from extracellular components of C. saccharoperbutylacetonicum (ATCC 13564) (11).

In this work, we hypothesize that the identification and deletion of autolysin genes cannot only help to sustain cell biomass and increase the butanol production but also increase the stability and recyclability of the culture cells and thus enhance the performance for long-term continuous fermentations. In this study, we identified the putative autolysin genes in C. saccharoperbutylacetonicum N1-4 based on the homologous analysis using BLAST. Then, we overexpressed all 16 putative autolysin genes individually in C. saccharoperbutylacetonicum and, by characterizing these recombinant strains, identified four key genes to be responsible for significant cell autolysis activities. Furthermore, we deleted these key autolysin genes using our customized CRISPR-Cas9 system. Further characterization of the mutants through fermentation revealed valuable insights into the effects of autolysins on cell stability and solvent production.

\section{RESULTS AND DISCUSSION}

Identification of the autolysin genes. Based on the information about autolysin genes available in the literature, the putative autolysin genes were identified in C. saccharoperbutylacetonicum N1-4 (Table 1), which were grouped into four families. It could be noticed that the muramidase-encoding genes in C. saccharoperbutylacetonicum account for most of the putative autolysin genes.

Figure 1 shows the growth curve of the 16 strains containing the putative autolysin gene. All the recombinant strains showed decreased growth compared to the control strain, suggesting that all the autolysin genes likely incurred the autolytic activity in the corresponding strain. The gray area on the profile represents the difference in cell growth between the recombinant strain and the control strain. The data were analyzed using repeated-measures analysis of variance (ANOVA). The $P$ value is shown on the top of each profile in Fig. 1. For easier comparison, the curves were split at $36 \mathrm{~h}$. For all the curves, no significant difference in the cell growth (the recombinant strain versus the control strain) was noticed during the exponential phase (up to $36 \mathrm{~h}$ ), but significant differences (at 90\% confidence level; $\alpha<0.1$ ) were observed in profiles (with the strains PJB24, PJB26, PJB29, and PJB30) during the stationary and death phases (after $36 \mathrm{~h}$ ). From the four genes, 24, 26, 29, and 30, two (gene 24 and gene 29) of them have 
TABLE 1 Sixteen putative autolysin genes identified in C. saccharoperbutylacetonicum N1-4

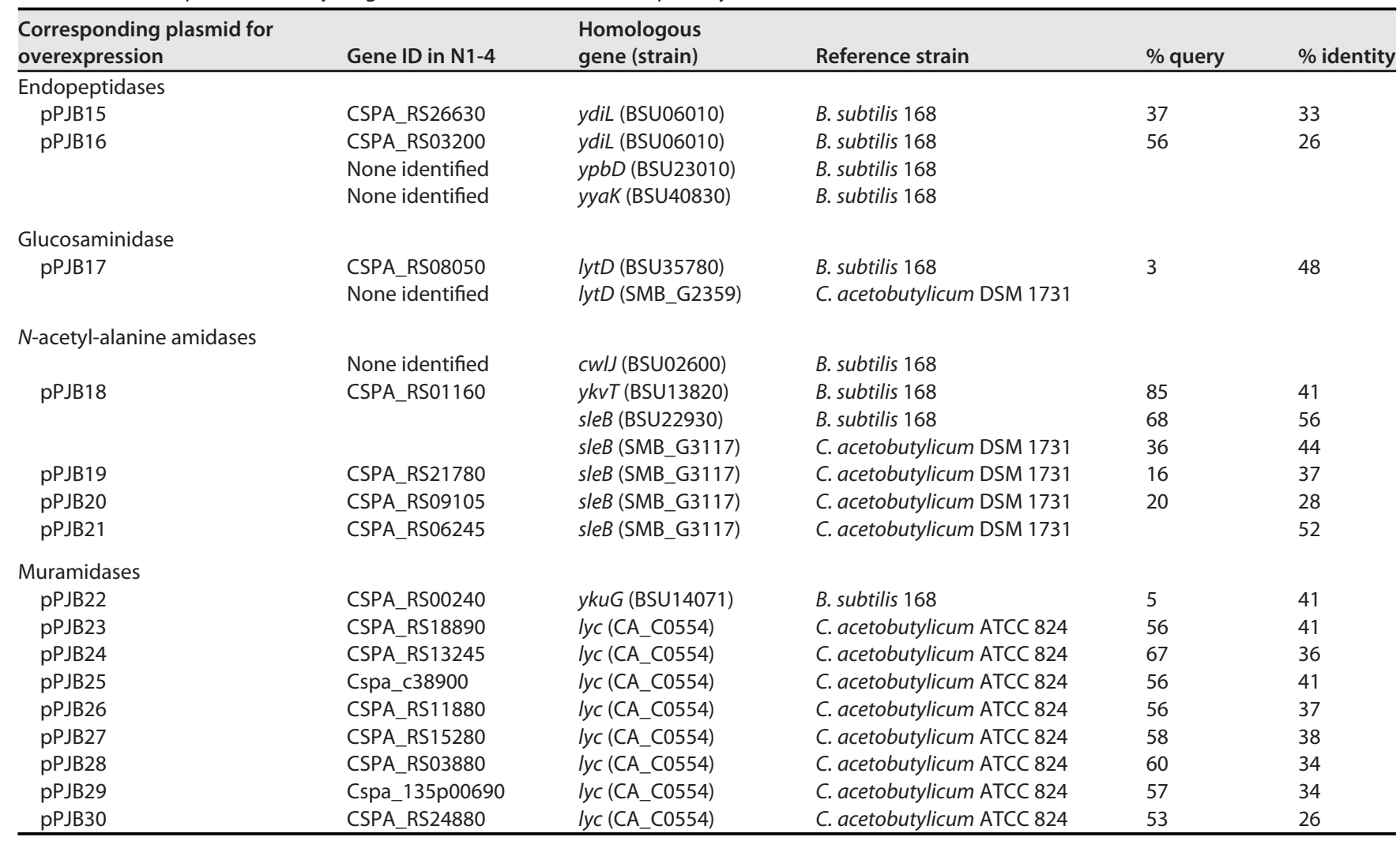

already been deleted in the prophage-deficient strains $\Delta 1234$ and $\Delta 12345$ prepared before (12). Therefore, we decided to delete the other two genes (gene 26 and gene 30 ) in N1-4 wild-type, $\Delta 1234$, and $\Delta 12345$ strains in order to further evaluate their function related to cell autolysis.

Recently, we obtained the N1-4-C strain by curing the endogenous megaplasmid of the N1-4 strain (13). N1-4-C could produce slightly higher solvents than the mother strain N1-4; however, a clear mechanism behind this was not identified. Based on the results here, the overexpression of gene 29 (Cspa_135p00690), which is located on the megaplasmid, could incur significant autolytic activity (Fig. 1). This might be the reason that N1-4-C could produce more solvents than N1-4 by eliminating this important autolysin gene through plasmid curing. On the other hand, gene 24 is contained in a putative prophage P2 (12) (see Table 2) on the N1-4 chromosome and is probably employed by the phage to enter into the lytic cycle.

Effects of the deletion of autolysin genes on butanol fermentation. Clean single deletion of gene 26 and gene 30 based on the three strains (N1-4, $\Delta 1234$, and $\Delta 12345$ ) was obtained (Fig. 2). The double deletion of both gene 26 and gene 30 in the same host was unsuccessful within any of these three mother strains despite numerous attempts. Although the reason behind this is not clear, autolysin genes are known to be related to essential metabolism such as cell division and sporulation $(6,14)$. This warrants further investigation in the future.

Small-scale batch fermentations were carried out in serum bottles. As shown in Fig. 3 , the deletion of gene 26 in N1-4 and $\Delta 12345$ led to increased production of cell biomass and reached a higher maximum optical density at $600 \mathrm{~nm}\left(\mathrm{OD}_{600}\right)$ than the mother strain (16.8 in $\Delta 26$ versus 14.6 in wild type and 18.8 in $\Delta 12345 \Delta 26$ versus 17.6 in $\Delta 12345)$. While in $\Delta 1234 \Delta 26$, the cell growth reached a similar maximum $\mathrm{OD}_{600}$ as the mother strain. In both $\Delta 1234 \Delta 26$ and $\Delta 12345 \Delta 26$, the $\mathrm{OD}_{600}$ remained at higher 


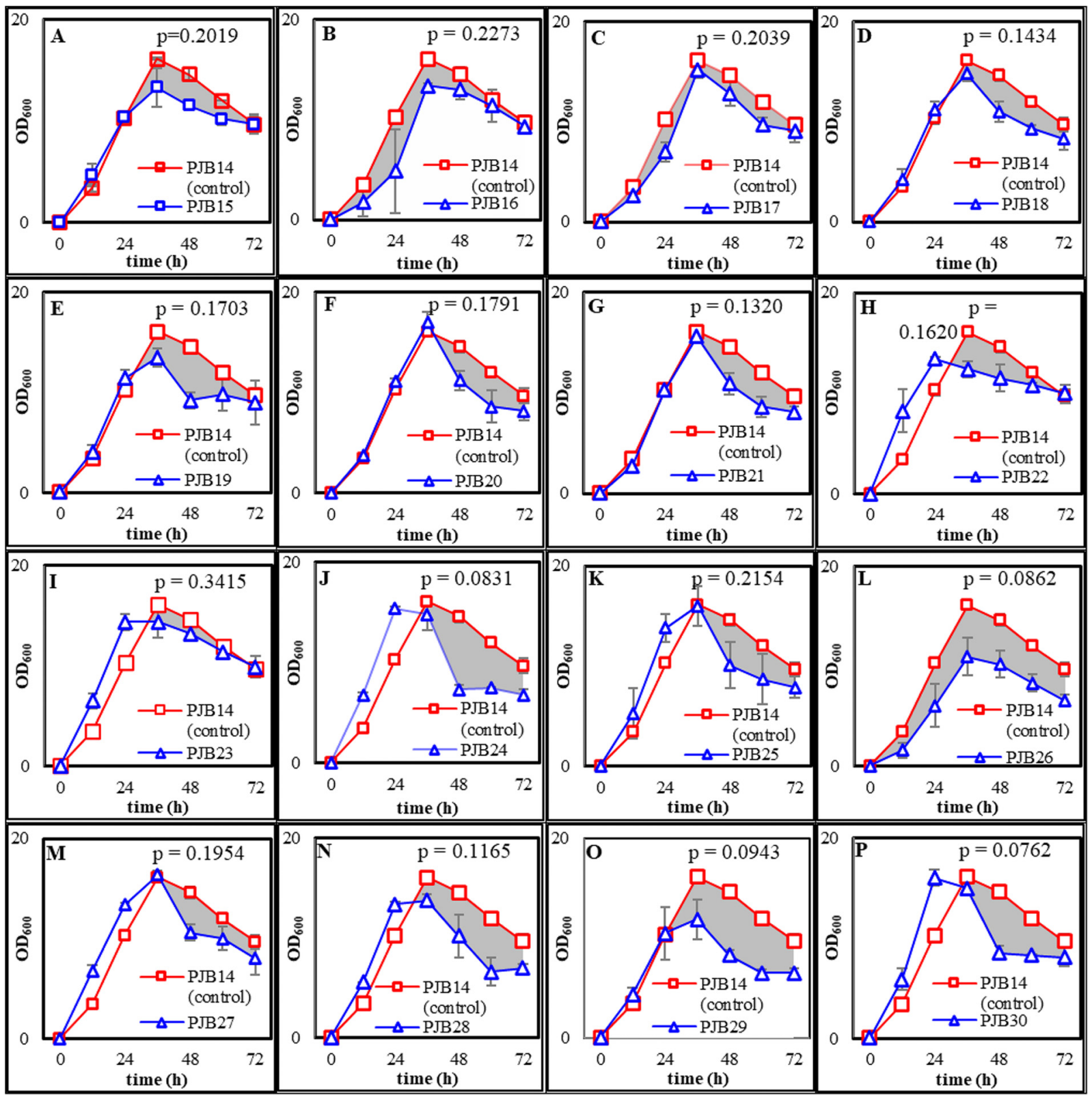

FIG 1 Growth profiles of the recombinant strains with overexpression of the putative autolysin genes compared to the control strain. The $P$ value at a $90 \%$ confidence level from the repeated-measures ANOVA during the stationary/death phases ( $36 \mathrm{~h}$ to $72 \mathrm{~h}$ ) has been put at the top of each profile. The error bar represents the standard error at a $95 \%$ confidence interval.

levels than their corresponding mother strains in the stationary/death phases, suggesting a reduction in autolysis with the deletion of these autolysis genes. This also confirmed the results that the overexpression of these autolysis genes resulted in a significant decline in cell biomass compared to the control during the stationary/death phases but not during the exponential phase (Fig. 1).

$\Delta 26$ produced slightly more butanol (but less acetone) than the wild type, while the butanol production in both $\Delta 1234 \Delta 26$ and $\Delta 12345 \Delta 26$ was at similar levels as the mother strains ( $\Delta 1234$ and $\Delta 12345$, respectively). On the other hand, the deletion of gene 30 in the wild type (generating the $\Delta 30$ strain) led to significantly decreased cell growth and acid crash in the fermentation with much lower solvent production. 
TABLE 2 Strains and plasmids used in this study

\begin{tabular}{|c|c|c|}
\hline Strain or plasmid & Description & Source or reference no. \\
\hline \multicolumn{3}{|l|}{ Strain } \\
\hline E. coli NEB Express & $\begin{array}{l}\left.\text { fhuA2 [lon] ompT gal sulA11 R(mcr-73:::miniTn10-Tet }{ }^{\mathrm{s}}\right) 2[\mathrm{dcm}] \mathrm{R}(\mathrm{zgb}-210:: \\
\left.\text { Tn10-Tet }{ }^{\mathrm{s}}\right) \text { endA1 } \Delta(\mathrm{mcrCmrr}) \text { 114::IS10 }\end{array}$ & New England Biolabs \\
\hline C. saccharoperbutylacetonicum N1-4 (HMT) & DSM 14923 (ATCC 27021), wild-type strain & DSMZ \\
\hline $\mathrm{N} 1-4-\mathrm{C}$ & $\begin{array}{l}\text { N1-4 strain } \Delta \text { Csp_135p (with the endogenous plasmid Csp_135p } \\
\text { eliminated) }\end{array}$ & 12 \\
\hline PJB14 & N1-4 harboring pPJB14 (empty plasmid) & This study \\
\hline PJB15 & N1-4 harboring pPJB15 & This study \\
\hline PJB16 & N1-4 harboring pPJB16 & This study \\
\hline PJB17 & N1-4 harboring pPJB17 & This study \\
\hline PJB18 & N1-4 harboring pPJB18 & This study \\
\hline PJB19 & N1-4 harboring pPJB19 & This study \\
\hline PJB20 & N1-4 harboring pPJB20 & This study \\
\hline PJB21 & N1-4 harboring pPJB21 & This study \\
\hline PJB22 & N1-4 harboring pPJB22 & This study \\
\hline PJB23 & N1-4 harboring pPJB23 & This study \\
\hline PJB24 & $\mathrm{N} 1-4$ harboring pPJB24 & This study \\
\hline PJB25 & N1-4 harboring pPJB25 & This study \\
\hline PJB26 & N1-4 harboring pPJB26 & This study \\
\hline PJB27 & N1-4 harboring pPJB27 & This study \\
\hline PJB28 & N1-4 harboring pPJB28 & This study \\
\hline PJB29 & N1-4 harboring pPJB29 & This study \\
\hline PJB30 & N1-4 harboring pPJB30 & This study \\
\hline$\Delta 26$ & N1-4 strain with CSPA_RS11880 deleted & This study \\
\hline$\Delta 30$ & N1-4 strain with the deletion of CSPA_RS24880 & This study \\
\hline$\Delta 1234$ & $\begin{array}{l}\text { N1-4-C with the deletion of the putative prophages P1, P2, P3, P4. P1 = } \\
\text { (Cspa_c09880-Cspa_c10360), P2=(Cspa_c26510-Cspa_c27350), P3= } \\
\text { (Cspa_c36410-Cspa_c36850), P4=(Cspa_c56920-Cspa_c57380) }\end{array}$ & 12 \\
\hline$\Delta 12345$ & $\begin{array}{l}\Delta 1234 \text { strain with the deletion of bacteriocin P5. P5=(Cspa_c07530- } \\
\text { Cspa_c07880) }\end{array}$ & 12 \\
\hline$\Delta 1234 \Delta 26$ & $\Delta($ CSPA_RS11880) & This study \\
\hline$\Delta 1234 \Delta 30$ & $\Delta\left(C S P A \_R S 24880\right)$ & This study \\
\hline$\Delta 12345 \Delta 26$ & $\Delta($ CSPA_RS11880) & This study \\
\hline$\Delta 12345 \Delta 30$ & $\Delta$ (CSPA_RS24880) & This study \\
\hline \multicolumn{3}{|l|}{ Plasmid } \\
\hline pJZ100 & Derived from TJ1, used for pPJB14 construction & 21 \\
\hline pPJB14 & Mother vector for gene overexpression under $P_{\text {lac }}$ & This study \\
\hline pPJB15 & pPJB14 derivative, for the overexpression of CSPA_RS26630 & This study \\
\hline pPJB16 & pPJB14 derivative, for the overexpression of CSPA_RS03200 & This study \\
\hline pPJB17 & pPJB14 derivative, for the overexpression of CSPA_RS08050 & This study \\
\hline pPJB18 & pPJB14 derivative, for the overexpression of CSPA_RS01160 & This study \\
\hline pPJB19 & pPJB14 derivative, for the overexpression of CSPA_RS21780 & This study \\
\hline pPJB20 & pPJB14 derivative, for the overexpression of CSPA_RS09105 & This study \\
\hline pPJB21 & pPJB14 derivative, for the overexpression of CSPA_RS06245 & This study \\
\hline pPJB22 & pPJB14 derivative, for the overexpression of CSPA_RS00240 & This study \\
\hline pPJB23 & pPJB14 derivative, for the overexpression of CSPA_RS18890 & This study \\
\hline pPJB24 & pPJB14 derivative, for the overexpression of CSPA_RS13245 & This study \\
\hline pPJB25 & pPJB14 derivative, for the overexpression of Cspa_c38900 & This study \\
\hline pPJB26 & pPJB14 derivative, for the overexpression of CSPA_RS11880 & This study \\
\hline pPJB27 & pPJB14 derivative, for the overexpression of CSPA_RS15280 & This study \\
\hline pPJB28 & pPJB14 derivative, for the overexpression of CSPA_RS03880 & This study \\
\hline pPJB29 & pPJB14 derivative, for the overexpression of Cspa_135p00690 & This study \\
\hline pPJB30 & pPJB14 derivative, for the overexpression of CSPA_RS24880 & This study \\
\hline pYW51 & CRISPR-Cas9 mother vector, used to construct plasmid for gene deletions & 22 \\
\hline pPJB31 & pYW51 derivative for CSPA_RS11880 deletion & This study \\
\hline pPJB32 & pYW51 derivative for CSPA_RS24880 deletion & This study \\
\hline
\end{tabular}

However, the deletion of gene 30 in either $\Delta 1234$ or $\Delta 12345$ did not result in a significant phenotype difference in the resultant mutants compared to their mother strains.

Further characterization with batch fermentations in bioreactors with $\mathrm{pH}$ control was performed for C. saccharoperbutylacetonicum N1-4, $\Delta 1234$, and $\Delta 1234 \Delta 26$. As 


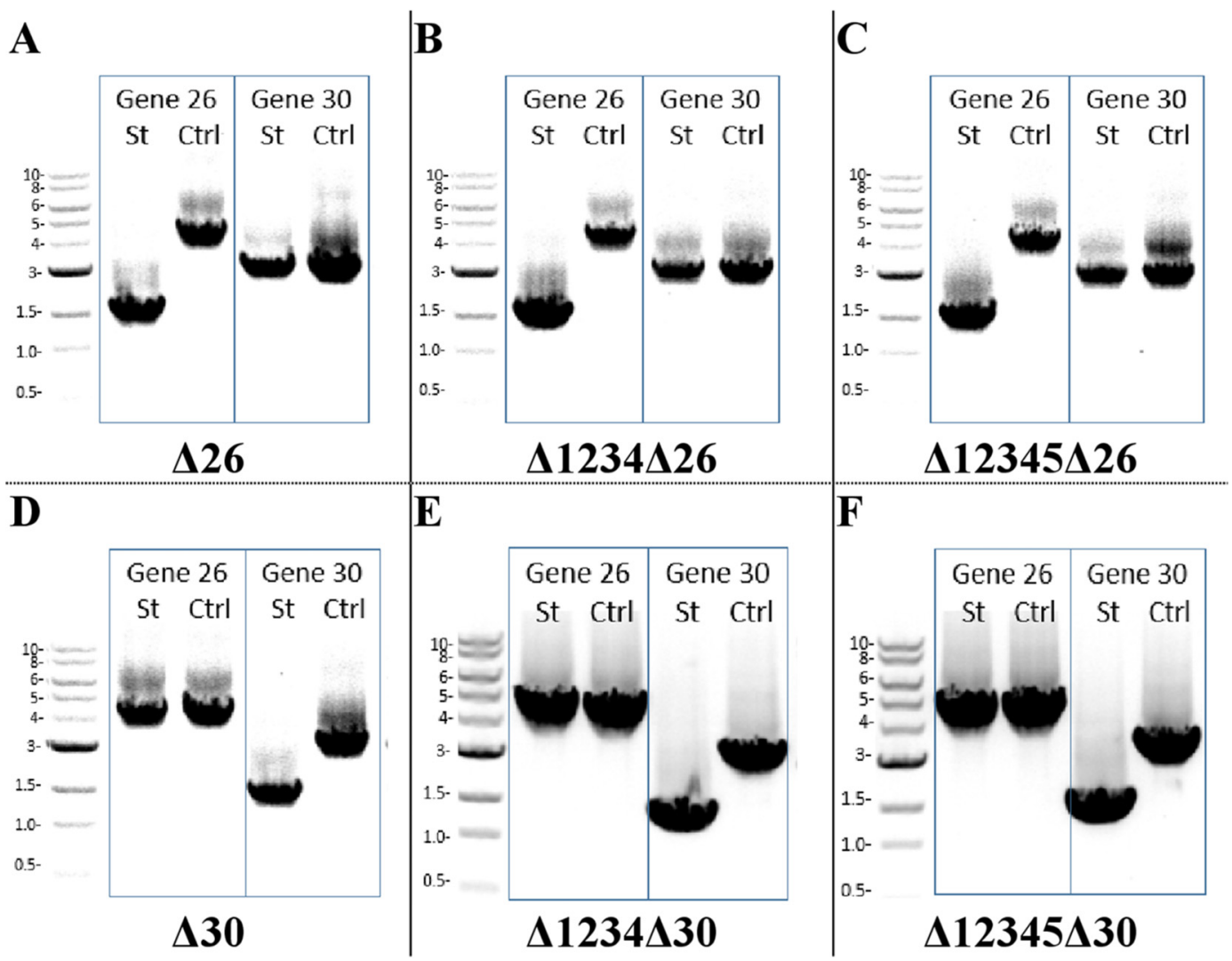

FIG 2 Agarose gel electrophoresis showed the colony PCR results confirming the gene deletion in the mutant strain (St) versus the control strain (Ctrl). (A) Deletion of gene 26 in N1-4; (B) deletion of gene 26 in $\Delta 1234$; (C) deletion of gene 26 in $\Delta 12345$; (D) deletion of gene 30 in N1-4; (E) deletion of gene 30 in $\Delta 1234$; (F) deletion of gene 30 in $\Delta 12345$. The positive mutant for the deletion of gene 26 should have a PCR band of 2,186 bp (versus 4,166 bp in the control), and the positive mutant for the deletion of gene 30 should have a PCR band of 2,125 bp (versus 3,070 bp in the control). The NEB $1-\mathrm{kb}$ DNA ladder was used as the marker, with numbers on the left representing the band length in kilobases.

shown in Fig. 4 , overall, $\Delta 1234$ and $\Delta 1234 \Delta 26$ showed very similar fermentation profiles. $\Delta 1234$ and $\Delta 1234 \Delta 26$ produced a similar level of butanol, which was slightly higher than the wild type ( $\Delta 1234$ and $\Delta 1234 \Delta 26$ also consumed slightly more glucose than the wild type). It took slightly longer for $\Delta 1234$ and $\Delta 1234 \Delta 26$ to reach the maximum levels for solvent production and cell growth as well compared to the wild type.

Based on the repeated-measures ANOVA (Table S1 in the supplemental material), during the bottle fermentation, $\Delta 26$ produced a very similar level of butanol as the wild type, while all the other strains (excluding $\Delta 30$ ) produced significantly higher butanol levels than the wild type. $\Delta 30$ produced significantly lower levels of solvents than the wild type and all the other strains because of the acid crash. For pH-controlled fermentation in the bioreactor, the butanol production in $\Delta 1234$ was significantly higher than the wild type, while the butanol production in $\Delta 1234 \Delta 26$ showed no significant difference from either the wild type or $\Delta 1234$.

Transformation efficiency. During our experiments, we noticed significantly higher transformation efficiency of the $\Delta 12345$ strain than either the wild type or the $\Delta 1234$ strains. As shown in Table 3, $\Delta 12345$ has 6.5 - to 15 -fold higher transformation efficiency than the wild type or $\Delta 1234$. Although more research is needed to completely understand this phenomenon, our results suggest that the deletion of the prophage genome $\mathrm{P} 5$, or the combined deletion of P5 along with P1, P2, P3, and P4, might have an effect of improving the DNA transformation efficiency of the strain (12). It warrants further investigation of the effect of the deletion of autolysin gene(s) on DNA transformation efficiency in future studies. 


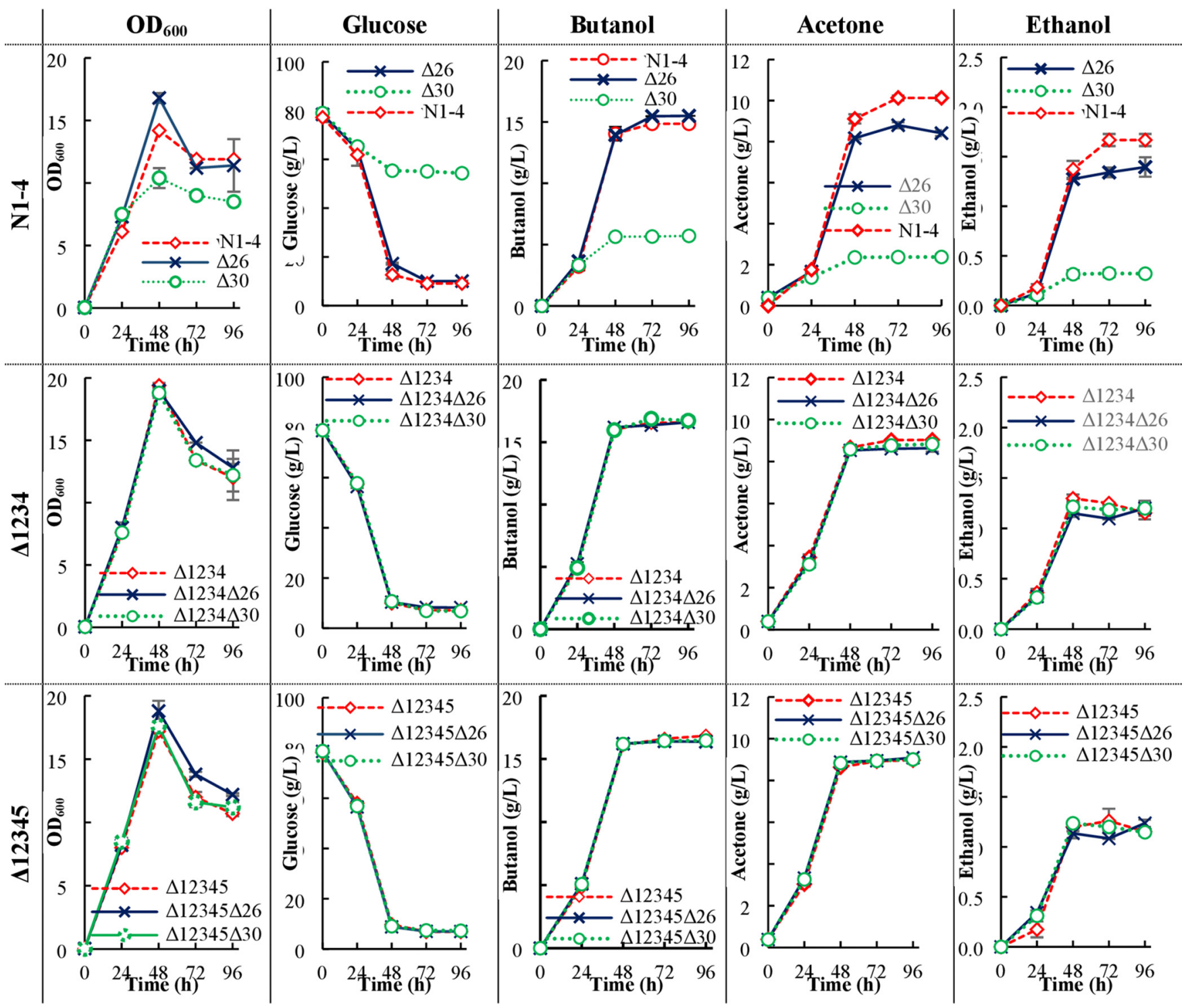

FIG 3 Profiles of serum bottle fermentation using the gene 26 and gene 30 single-deletion mutants compared to the mother strains ( $C$. saccharoperbutylacetonicum N1-4, $\Delta 1234$, and $\Delta 12345$, respectively). The error bar represents the standard error at a $95 \%$ confidence interval.

The development of the CRISPR-Cas9 system for genome editing in Clostridium has opened up broad possibilities for utilizing these strains as desirable platforms for biochemical production. However, Cas9 protein has high toxicity to the host cells due to its endonuclease activity; when it is expressed under a strong constitutive promoter, it is difficult to transform the CRISPR-Cas9 plasmid into the cells (no transformants could be obtained) (15). The expression of Cas9 under an inducible promoter is a feasible strategy to address this issue, as we do in our CRISPR-Cas9 system. In such a strategy, the genetic manipulation is achieved through homologous recombination first without the expression of Cas9, and then Cas9 is induced to express as a selection pressure for the positive mutant against the unedited mother cells (15). Even so, in many cases, the transformation efficiency is still low (compared to the control plasmid that does not contain Cas9) due to the leaked activity of the inducible promoter and thus toxicity of Cas9 to the cells. More complex systems, such as riboswitches, have been designed to reduce Cas9 toxicity during the transformation and increase expression during the selection for genome editing (16). However, such systems are often difficult to design and implement, and the efficiency is also varied. Alternatively, other researchers have utilized nickase-Cas9, which makes single-strand breakage and induces lower toxicity to the host, but such a strategy 


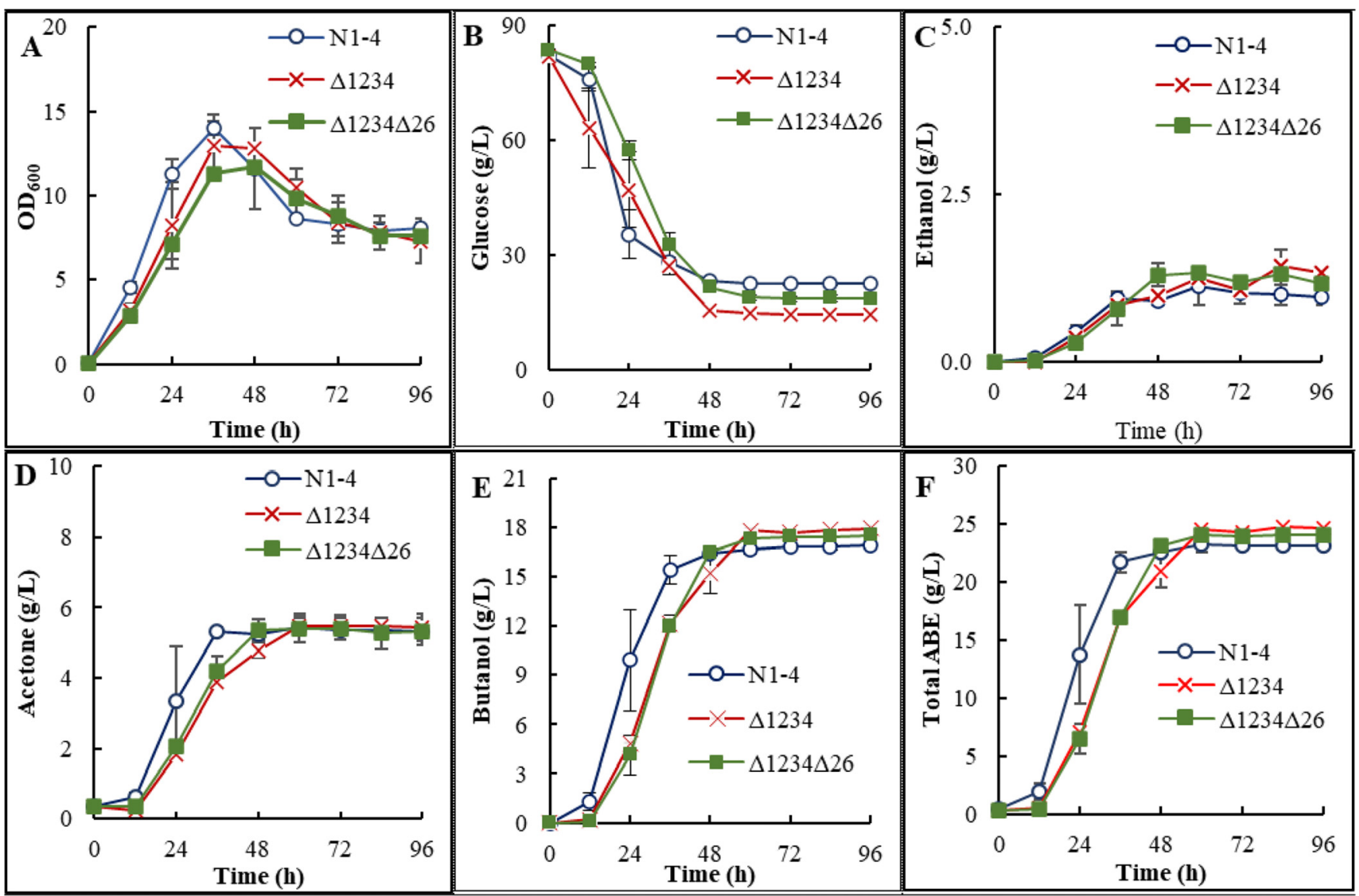

FIG 4 Profiles of batch fermentation in bioreactors with $\mathrm{pH}$ controlled for $\Delta 1234 \Delta 26$ compared to $\Delta 1234$ and the wild type (N1-4). The error bar represents the standard error at a $95 \%$ confidence interval.

would often sacrifice the selection efficiency of Cas9 (17). On the other hand, the Cas12a protein (Cpf1) makes double-strand breakage but with 5 bp distal, reducing the toxicity to some extent, and thus can also be used for genome editing with decent efficiency (18). In all cases, reliable transformation efficiency is still the key to success for genome editing in most Clostridium strains. Although in Clostridium saccharoperbutylacetonicum, the transformation efficiency is generally decently high, low transformation efficiency is still observed in some cases, for example, for the insertion of genes of large sizes using the CRISPR-Cas9 system. In a broader sense, the results we demonstrated here would provide an insightful reference for enhancing DNA transformation efficiency in other Clostridium strains and other microorganisms with underdeveloped DNA transformation protocols.

Effects of the deletion of autolysin genes on cell morphology. We checked the cell morphology of $\Delta 26$ and $\Delta 30$ compared to the wild-type mother strain (N1-4) under a microscope throughout the fermentation process. As shown in Fig. S1, at $96 \mathrm{~h}$ of the fermentation (row 2 in Fig. S1), when N1-4 (as well as $\Delta 30$ ) demonstrated obvious au-

TABLE 3 Transformation efficiency

\begin{tabular}{llll}
\hline & \multicolumn{3}{l}{ Transformation efficiency $(\mathrm{CFU} / \boldsymbol{\mu g})$ in host strain: } \\
\cline { 2 - 4 } Plasmid & Wild type & $\Delta \mathbf{1 2 3 4}$ & \multicolumn{1}{l}{$\mathbf{1 2 3 4 5}$} \\
\hline pPJB31 (N1-4) & 200 & 192 & 1,300 \\
pPJB32 (N1-4) & 101 & 271 & 1,500 \\
pPJB31 $(\Delta 30)$ & 236 & 329 & 1,100 \\
Avg $( \pm$ SE) & $179 \pm 57$ & $264 \pm 56$ & $1,300 \pm 163$ \\
\hline
\end{tabular}


tolysis, the cells of $\Delta 26$ were still in good clostridial shape with the regular sporulation. This is consistent with our results concerning the cell growth and solvent production: compared to N1-4, $\Delta 26$ grew to a higher maximum OD and produced slightly higher butanol level (Fig. 3), while $\Delta 30$ demonstrated acid crash and showed decreased cell growth compared to the wild type.

In this study, we identified the key autolysin genes responsible for the autolysis in the hyperbutanol-producing strain C. saccharoperbutylacetonicum N1-4, which provides an essential reference for the further development of robust strains for industrial fermentation. Peptidoglycan recycling is a metabolic process by which bacteria reutilize their cell wall biomass within one generation during vegetative growth. Peptidoglycan recycling has been extensively reported in Gram-negative bacteria (19). Recently, it has been revealed that peptidoglycan recycling also occurs in some Gram-positive bacteria; although it is not essential for the vegetative growth of Gram-positive bacteria, it provides a benefit for the long-term survival in the stationary phase (20). It warrants further investigation of the peptidoglycan turnover and recycling in C. saccharoperbutylacetonicum and other Clostridium strains.

\section{MATERIALS AND METHODS}

Reagents and strains. Phanta HS Super-Fidelity DNA polymerase and ClonExpress MultiS One Step cloning kit from Vazyme Biotech (Nanjing, China) were used for PCR cloning and plasmid construction through DNA assembly, respectively. Taq DNA polymerase from Green Mountains Biosystems (Ann Arbor, MI) and LongAmp Taq from New England BioLabs, Inc. (Ipswich, MA) were used for colony PCR (CPCR) to confirm the mutation. BtgZl and Notl restriction enzymes were obtained from New England BioLabs, Inc. All polymerases and restriction enzymes were used following the manufacturers' protocols.

All the strains used in this study are listed in Table 2. Escherichia coli NEB Express (New England BioLabs, Inc.) was used for plasmid propagation. E. coli was cultivated in Luria-Bertani (LB) broth or LB agar plates supplemented with $100 \mu \mathrm{g} / \mathrm{ml}$ carbenicillin when needed. C. saccharoperbutylacetonicum N1-4 (HMT) (DSM 14923) obtained from DSMZ (Braunschweig, Germany), and phage-deficient strains C. saccharoperbutylacetonicum $\Delta 1234$ and $\Delta 12345$ were routinely cultivated in tryptone-glucose-yeast extract (TGY) medium containing $30 \mathrm{~g} /$ liter of tryptone, $20 \mathrm{~g} /$ liter of sucrose, $10 \mathrm{~g} /$ liter of yeast extract, and $1 \mathrm{~g} /$ liter of L-cysteine, or on TGY agar plates, in an anaerobic chamber at $35^{\circ} \mathrm{C}$. We supplemented $30 \mu \mathrm{g} / \mathrm{ml}$ clarithromycin (Cla) and/or $40 \mathrm{mM}$ lactose (TGYL) when necessary. SMP buffer used for electrotransformation is composed of $270 \mathrm{mM}$ sucrose, $1 \mathrm{mM} \mathrm{MgCl}$, and $7 \mathrm{mM} \mathrm{Na} \mathrm{HPO}_{3} / \mathrm{NaH}_{2} \mathrm{PO}_{3}$ (pH adjusted to 6.5 and filter sterilized). Clostridium strains were stored at $-80^{\circ} \mathrm{C}$ in glycerol stocks (20\%). The stock was inoculated into TGY at $5 \%$ inoculum, and the culture was incubated at $35^{\circ} \mathrm{C}$ overnight to prepare the seed culture for DNA transformation or fermentation.

Plasmid construction. All the plasmids used in this study are listed in Table 2. All the primers used in this study are listed in Table 4. pJZ100 (21) was used as the mother vector to construct pPJB14. The $P_{\text {lac }}$ promoter was cloned from plasmid pYW51 using primers YW2583 and YW2584 and then inserted between the Apal and BtgZl sites of pJZ100 to replace the original $P_{\text {thl }}$ promoter, generating pPJB14.

Sixteen putative autolysin-encoding genes (CSPA_RS26630, CSPA_RS03200, CSPA_RS08050, CSPA_RS01160, CSPA_RS21780, CSPA_RS09105, CSPA_RS06245, CSPA_RS00240, CSPA_RS18890, CSPA_RS13245, Cspa_C38900, CSPA_RS11880, CSPA_RS15280, CSPA_RS03880, Cspa_135p00690, and CSPA_RS24880; numbered as autolysin gene 15 to 30, respectively) from C. saccharoperbutylacetonicum N1-4 were identified using the NCBI BLAST tool based on the homologous amino acid sequences from previously identified autolysin-encoding genes in related strains (5). The DNA fragment of the putative autolysin gene was cloned individually using PCR with the set of primers (Table 4) and the genomic DNA of the N1-4 strain as the template. pPJB14 was digested with $\mathrm{Btg} Z \mathrm{Zl}$, and then the DNA fragment of each autolysin gene was inserted to obtain the plasmid (pPJB15 to pPJB30; Table 2) for overexpression purposes.

Autolysin genes 26 and 30 (corresponding to plasmids pPJB26 and pPJB30, respectively) were selected to delete in C. saccharoperbutylacetonicum N1-4, $\Delta 1234$, and $\Delta 12345$ using our customized CRISPR-Cas9 genome editing system $(15,22)$. Plasmid pYW51 was used as the mother vector to construct the plasmid for gene deletion (Table 2). To delete gene 26, the small RNA (sRNA) (sCbei_5830) promoter fused with the 20-nucleotide $(\mathrm{nt})$ guiding sequence (5'-GACTCTCCATTAATAGTAATCC- $\left.3^{\prime}\right)$ was amplified from C. beijerinckii 8052 genomic DNA using primers YW5190 and YW484 and inserted into the BtgZl site of pYW51, generating an intermediate vector. Further, two homology arms ( $\sim 1 \mathrm{~kb}$ in length for each) flanking at both sides of the open reading frame (ORF) of gene 26 were amplified from C. saccharoperbutylacetonicum genomic DNA with primer pairs of YW5191/YW5247 and YW5193/YW5194, respectively, and then inserted into the Notl site of the intermediate vector mentioned above, generating pPJB31 for the deletion of gene 26. Similarly, for targeting on gene 30 for the gene deletion, the fragment containing the sRNA promoter and 20-nt guiding sequence (5'-ACTGGTATCCCATAACTTCT-3') was amplified with primers YW5201 and YW484 and then inserted into the BtgZl site of pYW51, generating an intermediate vector. One $\sim 1$-kb homology arm sequence upstream of gene 30 was amplified using primers YW5197/YW5249, and another $\sim 1-\mathrm{kb}$ homology arm sequence downstream of gene 30 was amplified using primers YW5250/ 
TABLE 4 List of primers used in this study

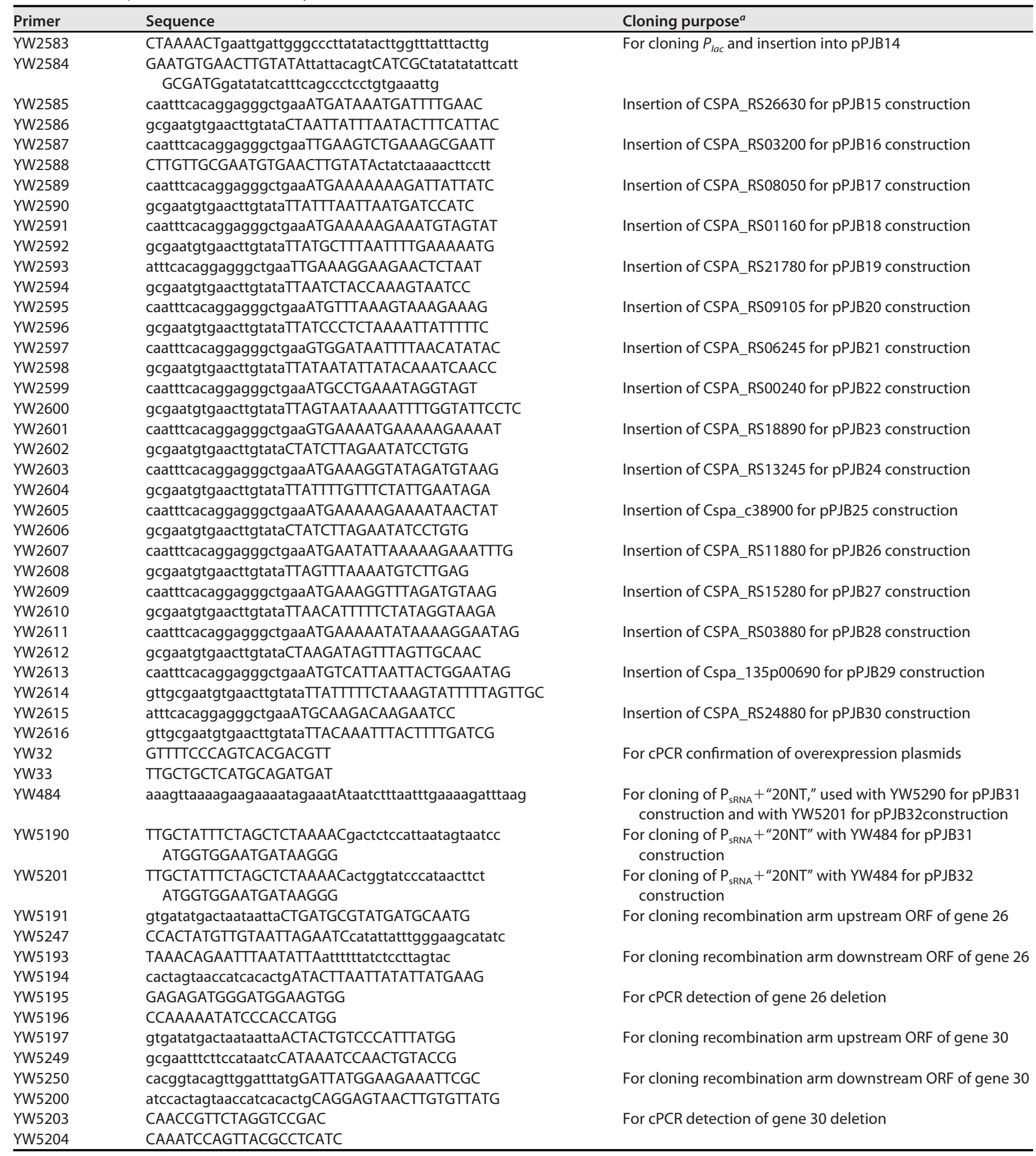

YW5200. The two homology arms were then inserted into the Notl site of the intermediate vector as mentioned above, generating PPJB32 for the deletion of gene 30.

Plasmid transformation and mutant screening. Clostridium cells were electroporated using our previously published protocol (22), with $2.0 \mathrm{kV}(1 \mathrm{kV} / \mathrm{mm}), 25 \mu \mathrm{F}$, and $300 \Omega$; SMP (instead of glycerol) was used as the washing buffer.

For the recombinant strain for gene overexpression (bearing plasmids pPJB15 to pPJB30, 
respectively), single colonies were picked for CPCR to confirm the existence of the plasmid using primers YW32/YW33. The confirmed positive recombinant strain was then inoculated into the TGY-Cla ${ }^{+}$liquid medium for cultivation. When the culture was grown to early exponential phase, glycerol stock was prepared for each culture and stored at $-80^{\circ} \mathrm{C}$ for later use. For obtaining the mutant strain with gene deletion (for the deletion of gene 26 or gene 30 ), single colonies from transformants were picked and inoculated into $2 \mathrm{ml} \mathrm{TGY} \mathrm{liquid} \mathrm{medium.} \mathrm{When} \mathrm{the} \mathrm{culture} \mathrm{grew} \mathrm{up} \mathrm{(at} \mathrm{the} \mathrm{exponential} \mathrm{phase),} 50 \mu \mathrm{l}$ was taken and spread onto $\mathrm{TGYL}^{-\mathrm{Cla}^{+}}$agar plates to induce the Cas9 activity for mutant selection. The mutation was confirmed using CPCR with primers flanking upstream and downstream of the targeted homologous recombination region (YW5195/YW5196 and YW5203/YW5204 for confirming the deletion of gene 26 and gene 30, respectively; Table 4). Then, the identified positive mutants were cultured and subcultured in liquid TGY (for 1 to 2 generations) for plasmid curing. The curing of the plasmid was confirmed through replica plating of the colonies onto both TGY and TGY-Cla ${ }^{+}$agar plates (the ones that could grow on the TGY plate but not on the TGY-Cla ${ }^{+}$plate were deemed the potential ones with plasmid cured). The mutation in the identified clean mutant (with plasmid cured) was further confirmed by CPCR.

Fermentation. Small-scale fermentations were carried out in 250-ml serum bottles with a working volume of $100 \mathrm{ml}$ for the nine strains N1-4, $\Delta 26, \Delta 30, \Delta 1234, \Delta 1234 \Delta 26, \Delta 1234 \Delta 30, \Delta 12345, \Delta 12345 \Delta 26$, and $\Delta 12345 \Delta 30$. The fermentation broth containing $80 \mathrm{~g} /$ liter glucose, $6 \mathrm{~g} /$ liter tryptone, and $2 \mathrm{~g} /$ /iter yeast extract in addition to the P2 medium was prepared and sterilized through autoclaving before starting the fermentation. P2 medium composition was reported before (23). The culture was grown in TGY medium in an anaerobic chamber at $35^{\circ} \mathrm{C}$ until the $\mathrm{OD}_{600}$ reached $\sim 0.8$. The culture was then inoculated at a $5 \%$ ratio (vol/vol) to the fermentation broth to initiate the fermentation. The fermentation in the serum bottles was carried out in an orbital shaker at $150 \mathrm{rpm}$ and $30^{\circ} \mathrm{C}$. Samples were taken every $24 \mathrm{~h}$ for analysis.

The strain harboring the plasmid for overexpression of the autolysin gene (pPJB15 to pPJB30, as shown in Table 1) was characterized through fermentation in 500-ml bioreactors (GS-MFC; Shanghai Gu Xin Biological Technology Co., Shanghai, China) under anaerobic conditions. The strain containing the empty plasmid (pPJB14) was used as the control. The fermentation broth containing $80 \mathrm{~g} /$ liter glucose, $6 \mathrm{~g} /$ liter tryptone, $2 \mathrm{~g} /$ liter yeast extract, and $13.7 \mathrm{~g} /$ liter lactose in addition to the P2 medium was prepared and autoclaved before the fermentation. We also supplemented $30 \mathrm{mg} /$ liter clarithromycin to the fermentation to help the culture maintain the plasmid. The fermentation was started with $5 \%$ inoculum from preculture (which was prepared by growing the strain in TGY medium supplemented with $30 \mathrm{mg} /$ /iter clarithromycin). The $\mathrm{pH}$ was controlled at $>5.0$ using $4 \mathrm{M} \mathrm{NaOH}$ throughout the fermentation. The temperature was kept at $30^{\circ} \mathrm{C}$. Samples were taken every $12 \mathrm{~h}$ for analysis. Each fermentation was carried out in at least two replicates.

Larger-scale batch fermentations were carried out in replicates in BioFlo 115 benchtop bioreactors (New Brunswick Scientific Co., Enfield, CT) with a working volume of 1.5 liters for $\Delta 1234$ and $\Delta 1234 \Delta 26$ (with the wild-type strain as the control). The fermentation broth containing $80 \mathrm{~g} /$ liter glucose, $6 \mathrm{~g} /$ liter tryptone, and $2 \mathrm{~g} /$ /iter yeast extract in addition to the $\mathrm{P} 2$ medium was prepared and sterilized before the fermentation. The fermentation was carried out at an agitation of $50 \mathrm{rpm}, 30^{\circ} \mathrm{C}$, with the $\mathrm{pH}$ controlled at $>5.0$ (using $4 \mathrm{M} \mathrm{NaOH}$ ). Samples were taken every $12 \mathrm{~h}$ for analysis.

Analytical procedures. Concentrations of glucose, butanol, acetone, and ethanol were determined on a high-performance liquid chromatographer (Agilent 1260 series; Agilent Technologies, Santa Clara, CA, USA) with a refraction index detector and Varian MetaCarb $87 \mathrm{H}$ column (set at $25^{\circ} \mathrm{C}$ ). Aqueous $5 \mathrm{mM}$ $\mathrm{H}_{2} \mathrm{SO}_{4}$ at a flow rate of $0.6 \mathrm{ml} / \mathrm{min}$ was used as the mobile phase. Cell optical density $\left(\mathrm{OD}_{600}\right)$ was quantified with a cell density meter (Ultrospec 10; Biochrom Ltd., Cambridge, England).

Microscopy. Cell culture was grown in P2 medium using $60 \mathrm{~g} /$ liter sucrose as the substrate. At specific time points, cell culture samples were harvested by centrifugation, washed twice, and resuspended in distilled water. Cell morphology was examined using an Olympus BX53F upright microscope (phase contrast mode) equipped with an Olympus DP73 camera (Olympus Corporation, Shinjuku-ku, Japan).

\section{SUPPLEMENTAL MATERIAL}

Supplemental material is available online only.

SUPPLEMENTAL FILE 1, PDF file, $0.1 \mathrm{MB}$.

\section{ACKNOWLEDGMENTS}

This work was supported by the Agriculture and Food Research Initiative competitive grant no. 2018-67021-27715 from the USDA National Institute of Food and Agriculture (NIFA), the USDA-NIFA Hatch Project (ALA014-1017025), the Ocean University of China-Auburn University (OUC-AU) grants program, and the Alabama Agricultural Experiment Station.

We thank Xeline Xia and Yu Gu for their assistance with the statistical analysis.

\section{REFERENCES}

1. Jiménez-Bonilla P, Wang Y. 2018. In situ biobutanol recovery from clostridial fermentations: a critical review. Crit Rev Biotechnol 38:469-482. https://doi.org/10.1080/07388551.2017.1376308.

2. Wang S, Dong S, Wang P, Tao Y, Wang Y. 2017. Genome editing in
Clostridium saccharoperbutylacetonicum N1-4 with the CRISPR-Cas9 system. Appl Environ Microbiol 83:e00233-17. https://doi.org/10.1128/AEM.00233 -17 .

3. Wang Y, Li X, Blaschek HP. 2013. Effects of supplementary butyrate on 
butanol production and the metabolic switch in Clostridium beijerinckii NCIMB 8052: genome-wide transcriptional analysis with RNA-Seq. Biotechnol Biofuels 6:138. https://doi.org/10.1186/1754-6834-6-138.

4. Al-Shorgani NKN, Shukor H, Abdeshahian P, Kalil MS, Yusoff WMW, Hamid AA. 2018. Enhanced butanol production by optimization of medium parameters using Clostridium acetobutylicum YM1. Saudi J Biol Sci 25:1308-1321. https://doi.org/10.1016/j.sjbs.2016.02.017.

5. Yang L, Bao G, Zhu Y, Dong H, Zhang Y, Li Y. 2013. Discovery of a novel gene involved in autolysis of Clostridium cells. Protein Cell 4:467-474. https://doi.org/10.1007/s13238-013-3025-x.

6. Smith TJ, Blackman SA, Foster SJ. 2000. Autolysins of Bacillus subtilis: multiple enzymes with multiple functions. Microbiology 146:249-262. https://doi.org/ 10.1099/00221287-146-2-249.

7. Liu Z, Qiao K, Tian L, Zhang Q, Liu Z-Y, Li F-L. 2015. Spontaneous largescale autolysis in Clostridium acetobutylicum contributes to generation of more spores. Front Microbiol 6:950. https://doi.org/10.3389/fmicb.2015 .00950 .

8. Branska B, Pechacova Z, Kolek J, Vasylkivska M, Patakova P. 2018. Flow cytometry analysis of Clostridium beijerinckii NRRL B-598 populations exhibiting different phenotypes induced by changes in cultivation conditions. Biotechnol Biofuels 11:99. https://doi.org/10.1186/s13068-018-1096-x.

9. Croux C, García J. 1991. Sequence of the lye gene encoding the autolytic lysozyme of Clostridium acetobutylicum ATCC824: comparison with other lytic enzymes. Gene 104:25-31. https://doi.org/10.1016/0378-1119(91)90460-S.

10. Croux C, Canard B, Goma G, Soucaille P. 1992. Purification and characterization of an extracellular muramidase of Clostridium acetobutylicum ATCC 824 that acts on non-N-acetylated peptidoglycan. Appl Environ Microbiol 58:1075-1081. https://doi.org/10.1128/AEM.58.4.1075-1081.1992.

11. Yoshino S, Ogata S, Hayashida S. 1982. Some properties of autolysin of Clostridium saccharoperbutylacetonicum. Agr Biol Chem 46:1243-1248. https://doi.org/10.1080/00021369.1982.10865242.

12. Feng J, Zhang J, Wang P, Jimenez-Bonilla P, Gu Y, Zhou J, Cao M, Shao Z, Borovok I, Huang H, Wang J. 2020. Renewable fatty acid ester production in Clostridium. BioRxiv https://doi.org/10.1101/2020.03.29.014746.

13. Gu Y, Feng J, Zhang Z-T, Wang S, Guo L, Wang Y, Wang Y. 2019. Curing the endogenous megaplasmid in Clostridium saccharoperbutylacetonicum N1-4 (HMT) using CRISPR-Cas9 and preliminary investigation of the role of the plasmid for the strain metabolism. Fuel 236:1559-1566. https://doi.org/10.1016/j.fuel.2018.09.030.
14. Cullin N, Merritt J, Kreth J. 2017. Beyond cell division: the ecological roles of autolysins in oral biofilm communities. Curr Oral Health Rep 4:14-21. https://doi.org/10.1007/s40496-017-0118-2.

15. Wang Y, Zhang Z-T, Seo S-O, Lynn P, Lu T, Jin Y-S, Blaschek HP. 2016. Bacterial genome editing with CRISPR-Cas9: deletion, integration, single nucleotide modification, and desirable "clean" mutant selection in Clostridium beijerinckii as an example. ACS Synth Biol 5:721-732. https://doi.org/ 10.1021/acssynbio.6b00060.

16. Cañadas IC, Groothuis D, Zygouropoulou M, Rodrigues R, Minton NP. 2019. RiboCas: a universal CRISPR-based editing tool for Clostridium. ACS Synth Biol 8:1379-1390. https://doi.org/10.1021/acssynbio.9b00075.

17. Xu T, Li Y, Shi Z, Hemme CL, Li Y, Zhu Y, Van Nostrand JD, He Z, Zhou J. 2015. Efficient genome editing in Clostridium cellulolyticum via CRISPRCas9 nickase. Appl Environ Microbiol 81:4423-4431. https://doi.org/10 .1128/AEM.00873-15.

18. Hong W, Zhang J, Cui G, Wang L, Wang Y. 2018. Multiplexed CRISPR-Cpf1mediated genome editing in Clostridium difficile toward the understanding of pathogenesis of C. difficile infection. ACS Synth Biol 7:1588-1600. https://doi.org/10.1021/acssynbio.8b00087.

19. Reith J, Mayer C. 2011. Peptidoglycan turnover and recycling in Grampositive bacteria. Appl Microbiol Biotechnol 92:1-11. https://doi.org/10 .1007/s00253-011-3486-x.

20. Borisova M, Gaupp R, Duckworth A, Schneider A, Dalügge D, Mühleck M, Deubel D, Unsleber S, Yu W, Muth G, Bischoff M, Götz F, Mayer C. 2016. Peptidoglycan recycling in Gram-positive bacteria is crucial for survival in stationary phase. mBio 7:e00923-16. https://doi.org/10.1128/mBio.00923-16.

21. Zhang J, Wang P, Wang X, Feng J, Sandhu HS, Wang Y. 2018. Enhancement of sucrose metabolism in Clostridium saccharoperbutylacetonicum N1-4 through metabolic engineering for improved acetone-butanolethanol (ABE) fermentation. Bioresour Technol 270:430-438. https://doi .org/10.1016/j.biortech.2018.09.059.

22. Zhang Z-T, Jiménez-Bonilla P, Seo S-O, Lu T, Jin Y-S, Blaschek HP, Wang Y. 2018. Bacterial genome editing with CRISPR-Cas9: taking Clostridium beijerinckii as an example, p 297-325. In Braman J (ed), Synthetic Biology, vol 1772. Humana Press, New York, NY.

23. Yao D, Dong S, Wang P, Chen T, Wang J, Yue Z-B, Wang Y. 2017. Robustness of Clostridium saccharoperbutylacetonicum for acetone-butanolethanol production: effects of lignocellulosic sugars and inhibitors. Fuel 208:549-557. https://doi.org/10.1016/j.fuel.2017.07.004. 